\title{
LATTICE HOMOMORPHISMS INDUCED BY GROUP HOMOMORPHISMS
}

\author{
DONALD G. HIGMAN ${ }^{1}$
}

Introduction. By a lattice homorphism of a group $G$ onto a group $G^{\prime}$ we mean a single-valued mapping $\phi$ of the lattice $L(G)$ of subgroups of $G$ onto the lattice $L\left(G^{\prime}\right)$ of subgroups of $G^{\prime}$, which preserves all unions and intersections, that is, which is subject to the conditions

1. $\left(U_{\nu} S_{\nu}\right) \phi=U_{\nu}\left(S_{\nu} \phi\right)$,

2. $\left(\bigcap_{\nu} S_{\nu}\right) \phi=\bigcap_{\nu}\left(S_{\nu} \phi\right)$,

for every (finite or infinite) set of subgroups $S_{\nu}$ of $G$. We call proper any lattice homomorphism which is neither a lattice isomorphism $(1-1)$, nor a trivial lattice homomorphism $(S \phi=1$ for every $S$ in $L(G)$ ). The general problem which now presents itself is the characterization of those (subgroup) lattice mappings which are proper lattice homomorphisms, and of those groups which admit proper lattice homomorphisms (cf. Whitman [1], ${ }^{2}$ Zappa [1] and [2], and Suzuki [1]).

A specialization of this problem arises when we consider a lattice mapping which is induced by a (group) homomorphism. It is easily seen that such a mapping preserves unions. Under what conditions will it be a (proper) lattice homomorphism? The main concern of this note is the characterization of those homomorphisms which induce proper lattice homomorphisms, and of those groups which admit such homomorphisms.

A further specialization occurs in connection with a method suggested by G. Zappa for constructing lattice mappings which preserve intersections. Let $H$ be a proper subgroup of a group $G$. Then the mapping $\psi$ of $L(G)$ onto $L(H)$ defined by

$$
S \psi=S \cap H \text { for each } S \text { in } L(G)
$$

clearly preserves intersections. In case $\psi$ is induced by an endomorphism of $G$, which maps $G$ onto $H$, it will be a proper lattice homomorphism of $G$ onto $H$. The special problem is to characterize those groups which admit endomorphisms which induce lattice mappings of the type $\psi$.

G. Zappa has formulated and solved these problems concerning

Presented to the Society, April 27, 1951; received by the editors June 12, 1950.

1 The author wishes to express his thanks to Professor Reinhold Baer for suggesting this problem to him, and for his very many helpful suggestions during this investigation.

2 Numbers in brackets refer to the bibliography at the end of the paper. 
lattice homomorphisms induced by (group) homomorphisms, under the hypothesis that the groups involved are finite. It is the purpose of this note to remove this restriction. Our general results are independent of any hypotheses other than those of the problems as stated above, and include the results of Zappa. For the special problem, Theorem (1.1) is seen to be a direct extension of the Zappa result (Zappa [2, p. 185]). For the main problem we obtain, as an application of our theorem (2.1), and under hypotheses which are weaker than actual finiteness, results which directly generalize those of Zappa (see Zappa [2, p. 191] and appendices A and B of this paper).

Notations. For a group $G, L(G)$ equals the lattice of all subgroups of $G$. For any set of subgroups $S_{\nu}$ of $G, \cup_{\nu} S_{\nu}$ equals the subgroup generated by the $S_{\nu}$, called the union of the $S_{\nu}$; and $\bigcap_{\nu} S_{\nu}$ equals the intersection of the $S_{v}$. Also, if $A$ and $B$ are subgroups of $G, A \cup B$ equals the union of $A$ and $B$, and $A \cap B$ equals the intersection of $A$ and $B$.

- If $H$ and $K$ are groups, $H \times K$ equals the direct product of $H$ and $K$. If there is an isomorphism of $H$ onto $K$, we write $H \cong K$.

$\{s\}$ equals the cyclic group generated by $s$.

1. The special problem. The following lemma is required for the proof of Theorem (1.1).

Lemma (1.1). Let $N, H$ be proper subgroups of a group $G$. Then the following conditions are equivalent:

1. $S=(S \cap N) \times(S \cap H)$ for every subgroup $S$ of $G$.

2. (a) $G=N H$, and $N \cap H=1$.

(b) $n$ is in the cyclic group $\{n h\}$ for every $n$ in $N, h$ in $H$.

3. (a) $G=N \times H$.

(b) the elements of $G$ have finite order, and the orders of the elements of $N$ are prime to the orders of the elements of $H$.

Proof. 1 implies 2, for 1 implies 2(a) trivially. Let $n$ be any element of $N, h$ of $H$. If $n$ or $h$ is equal to 1 , there is nothing to prove. Assume $n \neq 1, h \neq 1$. Then by 1 ,

$$
\{n h\}=(\{n h\} \cap N) \times(\{n h\} \cap H) .
$$

Hence $n h=n^{\prime} h^{\prime}$, with $n^{\prime}$ in $\{n h\} \cap N$, and $h^{\prime}$ in $\{n h\} \cap H$. But, since $N \cap H=1$, the expression of $n h$ as a product of an element of $N$ with an element of $H$ is unique. Hence $n=n^{\prime}, h=h^{\prime}$.

2 implies 3 . Assume condition 2 satisfied, and let $n \neq 1, h \neq 1$ be two elements of $N$ and $H$ respectively. (We have assumed $N \neq 1, H \neq 1$.) We have assumed that $n$ is in $\{n h\}$, and hence $h$ is in $\{n h\}$, so that $n$ and $h$ commute. It follows that $G=N \times H$. It also follows that $n$ 
and $h$ have finite relatively prime orders, for there is an integer $i$ such that $n=(n h)^{i}=n^{i} h^{i}$, so that $n^{1-i}=h^{i}$ is in $N \cap H=1$. Hence $n$ and $h$ have finite orders, say $a$ and $b$ respectively, such that $1-i$ $=i^{\prime} a$, and $i=i^{\prime \prime} b$. Hence $a$ and $b$ are relatively prime. Since $G=N$ $\times H$, every element of $G$ has finite order.

We show finally that 3 implies 1 . Assume condition 3 satisfied. Let $S$ be any subgroup of $G, s$ any element in $S$. Then $s$ may be expressed uniquely as a product $n h$, with $n$ in $N, h$ in $H$. We have merely to show that $n$ and $h$ are in $S$. Let $a$ and $b$ be the orders of $n$ and $h$ respectively. Then $a x+b y=1$, so that $s=s^{a x+b y}=s^{a x} s^{b y}$. Now $s^{a x}=(n h)^{a x}$ $=h^{a x}$ is in $S \cap H$, and $s^{b y}=n^{b y}$ is in $S \cap N$. Consequently, $n=n^{b y}$, and $h=h^{a x}$ are in $S$. q.e.d.

TheOREM (1.1). Let $H$ be a proper subgroup of a group $G$. Then there exists an endomorphism $\omega$ of $G$ such that

$$
S \omega=S \cap H \text { for every subgroup } S \text { of } G
$$

if and only if every element in $G$ has finite order, $H$ is a direct factor of $G$, and the orders of the elements in $H$ are prime to the orders of the elements in $G / H$.

Proof. Suppose $\omega$ is an endomorphism of $G$ such that $S \omega=S \cap H$ for each subgroup $S$ of $G$. Let $N$ be the kernel of $\omega$. Then $N \neq G$, since $H \neq 1$. From

$$
1=N \omega=N \cap H
$$

it follows that $\omega$ induces an an automorphism of $H$. Further, since

$$
S \omega=S \cap H=S \text { for every subgroup } S \text { of } H
$$

it follows that the automorphism of $H$ induced by $\omega$ leaves invarient every subgroup of $H$. Let $\tau$ be the product of $\omega$ with the inverse of this automorphism. Since $G \omega=H, \tau$ is an endomorphism of $G$ which maps $G$ onto $H$, and leaves fixed every element of $H$. Now, since $S \omega$ is contained in $H, S \omega=S \tau$, for each $S$ in $L(G)$. Since $G \tau=H$, and since $\tau$ is idempotent, $G=N H$. Now since $H \neq G, N \neq 1$. We have shown that $N \cap H=1$. If $n$ is in $N, h$ in $H$, then

$$
\{n h\} \omega=\{n h\} \tau=\{h\} .
$$

Hence $h$ is in $\{n h\}$, so that Lemma (1.1), 2, is satisfied.

If conversely the conditions of Theorem (1.1) are satisfied, then let $\omega$ be the (decomposition) endomorphism of $G$ which maps every element onto its $H$-component. It follows from Lemma (1.1), 1, that 


$$
S \omega=[(S \cap N) \times(S \cap H)] \omega=S \cap H . \quad \text { q.e.d. }
$$

CoRollaRy (1.1). If $H$ is a proper subgroup of a group $G$, and $\omega$ is an endomorphism of $G$ such that $S \omega=S \cap H$ for every subgroup $S$ of $G$, then $G=N \times H$, where $N$ is the kernel of $\omega$.

2. The main problem. In the following sections we investigate the conditions under which the mapping of $L(G)$ onto $L\left(G^{\prime}\right)$ induced by a (group) homomorphism $\omega$ of $G$ onto $G^{\prime}$ is a lattice homomorphism. Because, as is easily seen, every such mapping preserves unions, we need only be concerned with the conditions under which it preserves intersections. Let $N$ be the kernel of $\omega$. Without loss in generality, we may identify $G / N$ with $G^{\prime}, \omega$ with the natural homomorphism of $G$ onto $G / N$. We are thus led to the definition:

A group $G$ has property $(Z)$ if there is a normal subgroup $N$ of $G$ such that the natural homomorphism $\omega$ of $G$ onto $G / N$ induces a proper lattice homomorphism of $G$ onto $G / N$. We shall also say that the pair $N, G$ has property $(Z)$.

If $N=1, \omega$ is the identity automorphism of $G$, which naturally induces a lattice isomorphism. If $N=G, \omega$ induces a trivial lattice homomorphism of $G$. We assume throughout our discussion that $N \neq 1, G$. (Frequent use is made of this hypothesis in the proof of Theorem (2.1)).

LemMa (2.1). Let $N$ be a normal subgroup of a group $G$. Then the pair $N, G$ has property $(Z)$ if and only if

$$
X \cap \bigcap_{s \in X}\{s\} \text { is not empty for every coset } X \text { in } G / N .
$$

Proof. Let $N, G$ be a pair having property (Z), and let $\omega$ be the natural homomorphism of $G$ onto $G / N$. Then for $X$ a coset in $G / N,\{s\} \omega=\{X\}$ for each $s$ in $X$. But, since $\omega$ is assumed to induce a lattice homomorphism, we also have $\left(\cap_{s} \in X\{s\}\right) \omega=\{X\}$. Hence, there is an element $r$ in $\bigcap_{8} \in x\{s\}$ such that $r$ is in $X$.

Assume conversely that the pair $N, G$ satisfies the conditions of the lemma, and consider any set of subgroups $S_{\nu}$ of $G$. Since $\omega$ is single-valued, $\left(\bigcap_{\nu} S_{v}\right) \omega$ is contained in $\bigcap_{\nu}\left(S_{\nu} \omega\right)$. We must show that for each coset $X$ in $G / N$ there is an element in $\bigcap_{\nu} S_{\nu}$ whose image under $\omega$ is $X$, that is, which is in $X$. Now $X$ in $\bigcap_{\nu}\left(S_{\nu} \omega\right)$ implies that for each $\nu$ there is an $s_{\nu}$ in $S_{\nu}$ such that $s_{\nu}$ is in $X$. But we have assumed that given $s_{\nu}$ in $X$, there is $r$ in $X$ such that $r$ is in $\bigcap_{\nu}\left\{s_{\nu}\right\}$. Hence $r$ is in $\bigcap_{r} S_{r}$. q.e.d.

We now introduce some notation which will prove useful throughout our discussion. Let $N$ be a normal subgroup of a group $G$. We de- 
note by $\phi$ the set of all primes which occur as orders of elements in $G / N$, and by $K$ and $N^{\prime}$ the set of all elements of $G$ and of $N$ respectively whose orders have prime factors exclusively from $\phi$. We denote by $N^{\prime \prime}$ the set of all elements of $N$ whose orders have no prime factors from $\phi$. Let us notice that

$$
\text { for each } X \text { in } G / N \text {, the set } X \cap K \text { is not empty. }
$$

For, let $X$ be a coset in $G / N$, and let $s$ be an element of $G$ in $X$. Then we can write $s=s^{\prime} s^{\prime \prime}$, where $s^{\prime}$ and $s^{\prime \prime}$ are in $\{s\}$, the order of $s^{\prime}$ has prime factors exclusively from $\phi$, and the order of $s^{\prime \prime}$ has no prime factors from $\phi$. Now $N s^{\prime \prime}=N$, since the order of $s^{\prime \prime}$ is prime to the order of every coset in $G / N$. Hence $X=N s=N s^{\prime}$, so that $s^{\prime}$ is in $X \cap K$.

Finally, for each $p$ in $\phi$, we denote by $N_{p}$ the set consisting of all those elements of $N$ of order a power of $p$.

We now state the fundamental theorem of our discussion.

TheOREM (2.1). Let $N$ be a normal subgroup of a group $G$. Then the pair $N, G$ has property $(Z)$ if and only if the elements of $G$ have finite order, and for each prime $p$ in $\phi$,

(a) every element in $G$ of order a power of $p$ is in $C$, the centralizer of $N$ in $G$, and

(b) $N_{p}$ is contained in the cyclic group generated by any element in $G$, not in $N$, having order a power of $p$.

We establish the proof in a series of steps. First let $N, G$ be a pair which has property $(Z)$, and denote by $R$ the set of all elements $r$, each of which is in $X \cap \bigcap_{8} \in x\{s\}$ for some $X=N r$ in $G / N$. Consider any pair of elements $r$ in $R, n$ in $N$, and put $s=n r$. Then, by Lemma (2.1), $r$ is in the cyclic group $\{s\}$. Hence there is an integer $i$ such that $r=s^{i}$. Hence $n=s r^{-1}=s^{1-i}$, so that both $n$ and $r$ are in the cyclic group $\{s\}$, and hence commute. We have:

1. $R$ is contained in $C$, the centralizer of $N$ in $G$, and $G=N C$. Now $r=(n r)^{i}=n^{i} r^{i}$. Hence:

2. For every $r$ in $R, n$ in $N$, there is an integer $i$ such that $r^{1-i}=n^{i}$.

It follows that the elements of $G / N$ have finite order, for the elements of $R$ have finite order modulo $N, N \neq 1$, and every coset of $G / N$ contains an element of $R$. Suppose now that $X$ is in $G / N, r$ in $R \cap X$. If we assume that $X \neq N$, then $r \neq 1$, and $X$ has finite order, say $m>0$. Now $r$ is congruent to $r^{1+m}$ modulo $N$, hence there is an integer $i \neq 0$, such that $r=r^{(1+m) i}$, so that $r^{(1+m) i-1}=1$. But $(1+m) i$ $-1 \neq 0$, since $m>0$. Hence, since $R \cap N=1$, every element of $R$ has finite order. If $n \neq 1$ is in $N$, then, as above, there is an integer $i \neq 0$, 
such that $r^{1-i}=n^{i}$. Consequently the elements of $N$ have finite order. Finally, since $G=N R$, and $R$ is contained in $C$, we have:

3. The elements of $G$ have finite order.

Let now $X$ be a coset in $G / N$ of order (a power of) a prime $p$. Then there is an element $d$ in $X$ of order a power of $p$. Let $r$ be in $R \cap X$; then $r$ is in $\{d\}$, and hence $r$ has order a power of $p$. Conversely, if $r$ in $R$ has order a power of $p$, then the coset $N r$ in $G / N$ has order a power of $p$. Hence:

4. There is an element in $R$ of order a power of a prime $p$ if and only if $p$ is in $\phi$.

Let now $p$ be a prime in $\phi$, and suppose there is $n$ in $N$ of order a power of $p$. Let $r$ be an element in $R$ of order a power of $p$. Then $r$ is in $\{n r\}$, and hence $n$ is in $\{n r\}$. Thus $\{n r\}$ is a cyclic group of prime power order having $\{n\}$ as a subgroup. Hence the cyclic group generated by any element of $\{n r\}$ not in $\{n\}$ contains $n$. In particular, $n$ is in $\{r\}$.

Now suppose there is in $G$ an element $d$ of order a power of $p$ which is not in $N$. Let $r$ be in $R \cap N D$, then $r$ is in $\{d\}$, and hence has order a power of $p$. Suppose $d=n r, n$ in $N$. Then $n=d r^{-1}$ is in $\{d\}$, and hence $n$ has order a power of $p$. Hence, by the above, $n$ is in $\{r\}$, $n=r^{j}$, so that $d=n r=r^{j+1}$. Thus $d$ is in $\{r\}$, and since $d$ is in $N r, d$ is in $R$, and hence in $C$. It also follows, from the above, that $N_{p}$ is contained in $\{d\}$, hence:

5. Let $p$ be a prime in $\phi$. Then every element in $G$ of order a power of $p$ is in the centralizer $C$ of $N$ in $G$, and the cyclic group generated by any element of $G$, not in $N$, of order a power of $p$ contains $N_{p}$.

We have established the necessity of the conditions of Theorem (2.1). Assume now conversely that the conditions of this theorem are satisfied by a group $G$ and a normal subgroup $N$ of $G$. Let $X$ be any coset in $G / N, t$ an element in $X \cap K$ such that the number of prime factors of the order of $t$ is minimal for the elements of $X \cap K$. (We have shown that $X \cap K$ is not empty.) By Lemma (2.1), it will suffice to show that for each $n$ in $N, t$ is in the cyclic group $\{n t\}$.

We can write $t=t_{1} t_{2} \cdots t_{k}$, where $t_{i}$ has order a power of a prime $p_{i}$, and $t_{i}$ is in $\{t\}$, for $i=1,2, \cdots, k$. We notice that no $t_{i}$ is in $N$, for otherwise $t t_{i}^{-1} \equiv t \bmod N$, and the order of $t t_{i}^{-1}$ has fewer prime factors than the order of $t$. For each $n$ on $N$, we can write $n$ $=n_{0} n_{1} n_{2} \cdots n_{k}$, where $n_{i}$ is in $\{n\}$ for $i=0,1, \cdots, k$, the order of $n_{i}$ is a power of $p_{i}$ for $i=1,2, \cdots, k$, and the order of $n_{0}$ is prime to each of $p_{1}, p_{2}, \cdots, p_{k}$. Now, the primes $p_{i}$ are in $\phi$, since $t$ is in $K$. Hence Theorem (2.1), (b) implies that $n_{i}$ and $t_{i}$ are in the centralizer of $N$ in $G$, and (c) implies that $n_{i}$ is in $\{t\}$, for $i=1,2, \cdots, k$. Hence 
$n_{i}=t_{i}^{a}, n_{i} t_{i}=t_{i}^{a+1}$. Now $1+a$ is prime to the order of $t_{i}$ since $p_{i}$ must divide $a$. Hence $t_{i}$ is contained in $\left\{t_{i}^{a+1}\right\}=\left\{n_{i} t_{i}\right\}$. It follows that $\left\{n_{i} t_{i}\right\}=\left\{t_{i}\right\}$. Thus

$$
\{t\}=\left\{\prod_{i=1}^{k} t_{i}\right\}=\prod_{i=1}^{k}\{t\}=\prod_{i=1}^{k}\left\{n_{i} t_{i}\right\}=\prod_{i=1}^{k}\left\{n_{i} t\right\}
$$

and

$$
\{n t\}=\left\{n_{0}\right\} \times \prod_{i=1}^{k}\left\{n_{i} t\right\} .
$$

Hence $t$ is contained in $\{n t\}$.

The proof of Theorem (2.1) is now complete.

Let $N$ be a normal subgroup of a group $G$. Since clearly every coset in $G / N$ contains an element whose order has prime divisors exclusively from $\phi$, we have the following corollary.

Corollary (2.1). Condition (a) of Theorem (2.1) is equivalent to

$\left(\mathrm{a}^{\prime}\right) G=N C$, where $C$ is the centralizer of $N$ in $G$.

And we notice that for each prime $p$ in $\phi$, there is an element in $G$, not in $N$, of order a power of $p$. Hence Theorem (2.1), (b) implies that $N_{p}$ is a cyclic group. Clearly $N^{\prime}$ is the direct product of the $N_{p}$. We have now immediately the following corollary.

Corollary (2.2). Condition (b) of Theorem (2.1) is equivalent to

$\left(b^{\prime}\right)$ for each prime $p$ in $\phi$, every (cyclic) p-group in $G$ either contains, or is contained in, $N_{p}$.

Corollary (2.3). If the pair $N, G$ has property (Z), then for each $p$ in $\phi, N_{p}$ is a cyclic group, and $N^{\prime}$ is the direct product of the $N_{p}$, for all $p$ in $\phi$.

3. Two reduction theorems. We require the notion of direct product with amalgamated central subgroup. Let $U, V$ be two groups such that $U \cap V=S$ belongs to the center of $U$ and the center of $V$. Form the direct product $U \times V$, as the set of all pairs $(u, v)$, with $u$ in $U, v$ in $V$. Consider the set $W$ of all pairs of the form $\left(s, s^{-1}\right)$, with $s$ in $S . W$ is a subgroup of the center of $U \times V$, and hence is normal in $U \times V$. Hence we may form the group $(U \times V) / W$, which we call the direct product of $U$ and $V$ with amalgamated central subgroup $S$, and denote by $U \times{ }_{S} V$. If $U$ and $V$ are subgroups of a group $G$, then $G=U \times{ }_{S} V$ if and only if $G=U V, U \cap V=S$, and $u v=v u$ for every $u$ in $U, v$ in $V$. Note that $V$ is the centralizer of $U$ in $G$ if and only if $S$ is the center of $U$. 
We now state our first reduction theorem. $C$ denotes the centralizer of $N$ in $G$, and $Z$ denotes the center of $N$.

TheORem (3.1). Let $N$ be a normal subgroup of a group $G$. Then the pair $N, G$ satisfies property $(Z)$ if and only if

1. every element of $G$ has finite order,

2. there is a subgroup $M$ of $G$ such that $G=N \times_{z} M$,

3. the orders of the elements of $N / Z$ are prime to the orders of the elements of $G / N \cong M / Z$,

4. the pair $Z, C$ has property $(Z)$, or $Z=1$.

Proof. Assume that the pair $N, G$ has property $(Z)$. Condition 1 is immediate. And, by Corollary (2.1), $G=N C$, so that $G=N \times{ }_{z} C$, hence 2. Also, by the first isomorphism law, $G / N \cong C / Z$. Since $N=N^{\prime} N^{\prime \prime}$, and $N^{\prime}$ is contained in $Z, N^{\prime \prime}$ contains a set of representatives of the cosets in $N / Z$; and $G=N K$. Condition 3 follows, since the orders of the elements in $N^{\prime \prime}$ are prime to the orders of the elements in $K$. We have yet to show in case $z \neq 1$, that the pair $Z, C$ has property (Z). Clearly we need only concern ourselves with Theorem (2.1), (b). Since $G / N \cong C / Z$, the set of all primes which occur as orders of cosets of $G / N$ and $C / Z$ coincide. We have called this set $\phi$. Let $d$ be any element of $C$, not in $Z$, such that $d$ has order a power of a prime $p$ in $\phi$. Then $d$ is not in $N$, since $Z=N \cap C$, hence, condition (b) follows, since we have assumed that the pair $N, G$ has property (Z).

Assume now conversely that the pair $N, G$ satisfies the conditions of Theorem (2.1). We need only show that the pair $N, G$ satisfies conditions (a) and (b) of Theorem (2.1). Condition 2 implies that $G=N C$, and hence that $G / N \cong N / Z$, so that again the set $\phi$ is the same for the pair $N, G$ and the pair $Z, C$. Let $d$ be any element of $G$ with order a power of a prime $p$ in $\phi$. Now, $G=N C$ implies that $G / Z$ is the product of $C / Z$ with $N / Z$, and since, by 3 , the orders of the elements of $C / Z$ and $G / N \cong C / Z$ are relatively prime, the coset $Z d$ is in $C / Z$. Thus $d$ is in $C$, and hence (a) is satisfied. Because of this, and because of 4 , the pair $N, G$ satisfies (b). q.e.d.

By Theorem (3.1) we have reduced our problem to the study of pairs of groups $N, G$ such that the elements of $G$ have finite order, and $N$ is a subgroup of the center of $G$ (and hence is normal and abelian). It is clear that for such pairs, Theorem (2.1), (a) will always be satisfied.

Now let $N$ be a subgroup of the center of a group $G$, and consider the sets $\phi, K, N^{\prime}$ and $N^{\prime \prime}$ as defined in $\S 2$. Since $N$ is abelian, $N=N^{\prime}$ $\times N^{\prime \prime} . N^{\prime}$ and $N^{\prime \prime}$ are normal in $G$, since they are subgroups of the center of $G$. Hence we may form the group $G / N^{\prime \prime}$. Clearly $N / N^{\prime \prime}$ 
belongs to the center of $G / N^{\prime \prime}$. And we notice that

$$
G / N \cong \frac{G / N^{\prime \prime}}{N / N^{\prime \prime}}
$$

Hence the set $\phi$ is exactly the set of primes which occur as orders of elements of the latter group. The orders of the elements of $N^{\prime \prime}$ are prime to the orders of the elements of $G / N^{\prime \prime}$, since $G=N^{\prime \prime} K$. Notice finally that, since $N / N^{\prime \prime} \cong N^{\prime}$, the existence in $N / N^{\prime \prime}$ of an element of prime order $p$ implies the existence in $G / N^{\prime \prime} / N / N^{\prime \prime}$ of an element of order $p$. Our second reduction theorem may now be stated as follows.

THEOREM (3.2). Let $N$ be a subgroup of the center of a group $G$, all of whose elements have finite order. Then the pair $N, G$ has property $(Z)$ if and only if the pair $N / N^{\prime \prime}, G / N^{\prime \prime}$ has property (Z), or $N=N^{\prime \prime}$.

Proof. Again we work with Theorem (2.1). Clearly we need only be concerned with condition (b), and the case $N \neq N^{\prime \prime}$.

First assume that the pair $N / N^{\prime \prime}, G / N^{\prime \prime}$ has property (Z). Let $n$ in $N$, and $d$ in $G, d$ not in $N$, each have order a power of a prime $p$ in $\phi$. Then $N^{\prime \prime} n$ is in $N / N^{\prime \prime}$, and $N d$ is in $G / N^{\prime \prime}, N d$ not in $N / N^{\prime \prime}$, and each of these cosets has order a power of $p$. Hence, by Theorem (2.1), (b), $N^{\prime \prime} n$ is contained in $\left\{N^{\prime \prime} d\right\}$. Hence $n \equiv d^{k} \bmod N^{\prime \prime}$ for some $k$, that is, $n=n^{\prime \prime} d^{k}$, with $n^{\prime \prime}$ in $N^{\prime \prime}$. But, since the order of $d$ is a power of a prime, and the orders of the elements of $N^{\prime \prime}$ are prime to the order of the coset $N d$, the order of $n^{\prime \prime}$ is prime to the order of $d$. Thus, since $n^{\prime \prime}$ and $d^{k}$ commute, $n^{\prime \prime}=1$, and hence $n$ is in $\{d\}$.

Assume conversely that the pair $N, G$ has property $(Z)$. Then let $X, Y$ be two elements of $G / N^{\prime \prime}$ such that $X$ is in $N / N^{\prime \prime}, Y$ is not in $N / N^{\prime \prime}$, and $X$ and $Y$ have orders a power of a prime $p$ in $\phi$. Then there exists $x$ in $X, y$ in $Y$ each having order a power of $p$. Thus, by Theorem (2.1), (b), $x$ is in $\{y\}$. This implies that $X$ is in $\{Y\}$. q.e.d.

By means of Theorems (3.1) and (3.2) we have reduced our problem to the study of pairs $N, G$ such that

$\left(^{*}\right) N$ is in the center of $G$, and the existence in $N$ of an element of prime order $p$ implies the existence in $G / N$ of a coset of order $p$.

If we now extend the definition of $N_{p}$ to all primes $p$ by setting $N_{p}=1$ for every $p$ not in $\phi$, wé have immediately, by Theorem (2.1) and Corollary (2.2), the following theorem.

TheOREм (3.3). Let $N$, G be a pair of groups satisfying the condition $\left(^{*}\right)$ as above. Then $N, G$ has property $(Z)$ if and only if the elements of 
$G$ have finite order, and for each prime p, every (cyclic) p-group in $G$ contains, or is contained in, $N_{p}$.

We also note that for a pair $N, G$ which satisfies condition (*), Corollary (2.3) reads as follows.

Corollary (3.1). For each prime $p, N_{p}$ is cyclic, and $N$ is the direct product of the $N_{p}$.

The construction problem which has now presented itself is to give a survey of all those pairs $N, G$ which satisfy the conditions and hypotheses of Theorem (3.3).

Appendix A. Zappa has obtained, for a finite group which has property $(Z)$, a decomposition which we now obtain under the somewhat weaker finiteness hypothesis $\left(\mathrm{F}^{\prime}\right)$ stated below, by application of the following well known result which is essentially due to Schur (cf. Zassenhaus [1, p. 125, Satz 24]).

Let an abelian group $U$ be a normal subgroup of a group $V$, and assume that $V / U$ has finite order. Then if the order of each element of $U$ is prime to the order of $V / U, V$ splits over $U$.

Let us assume now that $N, G$ is a pair which has property (Z), and further, that the finiteness condition,

$$
G / Z \text { is finite, where } Z \text { is the center of } N \text {, }
$$

is satisfied. Since $N^{\prime}$ is a subgroup of $Z$ (Theorem (2.1), (a) and Corollary (2.3)) and $Z$ is abelian, $Z=N^{\prime} \times T$, where $T$ is the subgroup of $Z$ consisting of those elements whose orders are prime to the orders of the elements in $N^{\prime}$. Consider now $\bar{N}=N / T$, and $\bar{Z}=Z / T$. Clearly $\bar{Z}$ is abelian, and $\bar{N} / \bar{Z}$ has finite order prime to the orders of the elements of $\bar{Z}$. Hence, by the above theorem of Schur, there is a subgroup $S$ of $G$ which contains $T$, and is contained in $N$, such that $\bar{N}=\bar{Z} \times S / T$, and hence $N=N^{\prime} \times S$. Now $S$ consists of the set of all elements of $N$ whose orders are prime to the orders of the elements of $N^{\prime}$, so that $S=N^{\prime \prime}$, and $N^{\prime \prime}$ is a characteristic subgroup of $N$. It follows, then, that $N^{\prime \prime}$ is a normal subgroup of $G$, and hence we may form the group $G / N^{\prime \prime}$. This group has finite order, since $N^{\prime}$ has finite order $\left(\left(F^{\prime}\right)\right.$ implies that the number of primes in $\phi$ is finite) and we have assumed that $G / Z$ is finite. Since property $(Z)$ implies $G=N C$, $G / N^{\prime \prime} \cong C /\left(Z \cap N^{\prime \prime}\right)$; and the orders of the elements of $Z \cap N^{\prime \prime}$ are prime to the orders of the elements of $C /\left(Z \cap N^{\prime \prime}\right)$. Thus, since $Z \cap N^{\prime \prime}$ is abelian, an application of Schur's theorem gives $C=T \times\left(Z \cap N^{\prime \prime}\right)$, and hence $G=T \times N^{\prime \prime}$, where $T$ consists of all those elements of $G$ whose orders are prime to the orders of the elements of $N^{\prime \prime}$, so that 
$T=K$. We have finally that

$$
G=N^{\prime \prime} \times K
$$

where $N^{\prime \prime}$ is the subgroup of $N$ consisting of all those elements of $N$ whose orders have no prime factors from $\phi$, and $K$ is the subgroup of $G$ consisting of all those elements of $G$ whose orders have prime factors exclusively from $\phi . \phi$, we recall, is the set of all primes which occur as orders of cosets of $G / N$.

Appendix B. In this second appendix we give a characterization of certain $p$-components of a group $G$ having property $(Z)$ which is analogous to the characterization of certain. Sylow subgroups found by Zappa in the finite case. Again we require a finiteness hypothesis which is weaker than actual finiteness.

Let $N, G$ be a pair which has property $(Z)$. Then, by Theorem (2.1), for each $p$ in $\phi$ such that $N_{p} \neq 1$, every $p$-component $P_{p}$ of $G$ contains $N_{p}$ as a proper subgroup, and every subgroup of $P_{p}$ either contains, or is contained in, $N_{p}$. Hence (cf. Baer $\left[1\right.$, p. 827]), if $P_{p}$ also satisfies the finiteness condition,

$\left(\mathrm{F}^{\prime \prime}\right)$ every finite set of elements in $P_{p}$ generates a finite group, then $P_{p}$ must have one of the following structures.

(i) if $p$ is odd, or if $P_{p}$ is abelian, then $P_{p}$ is a cyclic group of order a power of $p$, or $P_{p}$ is a group of type $p^{\infty}$. A group of type $p$ is an abelian group generated by elements $a_{1}, a_{2}, \cdots$ such that $a_{1}$ has order $p$, and $a_{i}^{p}=a_{i-1}$.

(ii) if $p=2$, and $P_{p}$ is not abelian, then $P_{p}$ is a dihedral group, that is, is generated by elements $u, v$ subject to the relations

$$
u^{2^{n+1}}=1, \quad u^{2^{n}}=v^{2}, \quad v u v^{-1}=u^{-1},
$$

or $P_{p}$ is an infinite dihedral group, that is, is generated by elements $u_{1}, u_{2}, \cdots, v$ such that

$$
u_{i}^{2}=u_{i-1}, \quad u_{1}^{2}=v^{2}, \quad u_{1}^{4}=1, \quad v u_{i} v^{-1}=u_{i}^{-1} .
$$

REMARK. We have not been able to ascertain whether or not the conditions $\left(\mathrm{F}^{\prime}\right)$ and $\left(\mathrm{F}^{\prime \prime}\right)$ are necessary for the results of the appendices. Evidently what is needed to extend the result of appendix A to the general case is an extension of the theorem of Schur.

\section{BIBLIOGRAPHY}

R. BAER

1. Duality and commutativity of groups, Duke Math. J. vol. 5 (1939) pp. 824-838. M. SuzukI

1. On the L-homomorphisms of finite groups, Trans. Amer. Math. Soc. vol. 70 (1951) pp. 372-386. 
P. M. Whitman

1. Groups with a cyclic group as lattice homomorph, Ann. of Math. vol. 49 (1948) pp. 347-351.

G. ZAPPA

1. Determinazione dei gruppi finiti in omomorfismo strutturale con un gruppo ciclico, Rendiconti del Seminario Matematico della Università di Padova vol. 18 (1949) pp. 140-162.

2. Sulla condizione perchè un omomorfismo ordinario sia anche un omomorfismo strutturale, Giornale di Matematiche di Battaglini (4) vol. 78 (1949) pp. 182-192.

H. ZASSENHAUS

1. Lehrbuch der gruppentheorie, 1937.

UNIVERSITY OF ILLINOIS

\section{SEMI-AUTOMORPHISMS OF SYMMETRIC AND ALTERNATING GROUPS}

\section{FLORA DINKINES}

1. Introduction. In 1947, Irving Kaplansky [5 $]^{1}$ extended certain results of Ancochea [1] on semi-automorphisms of division algebras by redefining the concept of a ring semi-automorphism.

The definition which proved fruitful there is: A semi-automorphism of a ring is an additive automorphism $a \rightarrow a^{\prime}$ satisfying $(a b a)^{\prime}=a^{\prime} b^{\prime} a^{\prime}$. It has been established that every semi-automorphism of a division ring is an automorphism or an anti-automorphism, [5] and [4], and this result has found application in various places; for example, in [2]. In view of this application of ring semi-automorphisms it is not inconceivable that a similar type of mapping for groups may prove useful. Following the ring concept we define a semi-automorphism of a group to be a 1-1 mapping of the group onto itself such that $(a b a)^{\prime}$ $=a^{\prime} b^{\prime} a^{\prime}$ and investigate the question: Under what conditions is a group semi-automorphism an automorphism or an anti-automorphism?

This article is an excerpt from a thesis prepared under the direction of Professor Kaplansky which answers this question for the following classical cases: symmetric groups, alternating groups, dihedral groups, finitely generated abelian groups, and certain linear groups over a 2-dimensional vector space.

For brevity we shall say that a group $G$ is SA if every semi-automorphism of $G$ is an automorphism or an anti-automorphism. For

Received by the editors July 25,1950 .

${ }^{1}$ Numbers in brackets refer to the bibliography at the end of the paper. 